\title{
Nodal Pricing of Power Balance Control Services in Electrical Power Systems
}

A. Jokic

\begin{abstract}
In this paper we formulate the problem of economically optimal and reliable spatial distribution of power balance ancillary services in electrical power networks. Furthermore, we propose a novel price-based (market-based) approach for scheduling of the control services in which we explicitly take into account limited capacity of transmission network. A novel notion of nodal pricing for power balance control services is introduced while constructive solution for calculation of nodal prices is presented.
\end{abstract}

\section{INTRODUCTION}

The power balancing Ancillary (control) Services (AS) markets are forward time markets ${ }^{1}$ whose goal is to cope with uncertainties that are inherently present in forward time decision making actions. The related forward time decisions considered in this paper are energy balancing actions, that is, balancing the power system "on paper", which takes place before the actual operation. The energy balancing is achieved by market clearing (finding equilibrium of demand and supply) in forward time energy markets. After the energy markets are cleared, the AS market clearing takes place. The quantities traded on the AS markets are options to deliver/retrieve additional energy within some predefined time interval if needed. In real-time, these options are called into operation by the corresponding transmission system operator (TSO) via appropriately designed feedback control loops, the so-called secondary control loops [1]. Forward time AS markets are aimed to ensure enough controllable prosumption (production/consumption) units that will be committed to act as actuators in the secondary control feedback loops. Loosely speaking, secondary control loops are responsible to cover for the "gaps" in between forward time made plans and real-time situation, while purchasing AS in forward time has to ensure that the saturation limits of the secondary control loops will be sufficiently large to cope with the expected sizes of these "gaps".

In [2], the author investigated interactions between reserves and energy and showed the necessity to consider not only energy but also reserves by location. In the current practice, the congestion management schemes have no guarantees that some lines in the system will not become congested

A. Jokic is with the Faculty of Mechanical Engineering and Naval Architecture, University of Zagreb, Ivana Lučića 5, 10000 Zagreb, Croatia, e-mail: andrej.jokic@fsb.hr.

P.P.J. van den Bosch is with the Department of Electrical Engineering, Eindhoven University of Technology, P.O. Box 513, 5600 MB Eindhoven, The Netherlands, e-mail: p.p.j.v.d.boschetue.nl.

${ }^{1}$ Here by forward time markets we mean markets in which commodities traded at time instant $t$ are to be delivered in some future time $t+\tau$, with $\tau>0$.
P.P.J. van den Bosch during provision of ancillary services. In other words, spatial dimension of AS is still an unsolved problem which has been recognized as such in the recent reports of European Network of Transmission System Operators [3], [4]. The problem formulated and solved in this paper is in line with the above mentioned needs. The contributions of this paper are summarized as follows: 1) problem formulation of pricebased (market-based), economically optimal and reliable spatial distribution of power balancing control services; 2) introduction of notion of nodal prices for the control services; 3) mathematical characterization of optimal nodal prices for control services, which is constructive. The obtained results are in particular important for the future power systems with large amount of uncontrollable renewable energy sources, which will be characterized by increase of uncertainties in any forward-time energy prosumption planning, while controllable power will become more scarce [5].

\section{NOTATION AND PRELIMINARIES}

1) Notation: We use $\mathbf{1}_{n}$ and $\mathbf{0}_{n}$ to denote a column vector with $n$ elements all being equal to 1 , and a column vector with $n$ elements all being equal to 0 , respectively. Let $c_{1}, \ldots, c_{n}$ be matrices / vectors with the same number of columns, or scalars, then $\operatorname{col}\left(c_{1}, \ldots, c_{n}\right):=$ $\left(\begin{array}{lll}c_{1}^{\top} & \ldots & c_{n}^{\top}\end{array}\right)^{\top}$. The convex hull of a set $\mathcal{C}$ is denoted by $\operatorname{conv} \mathcal{C}$.

2) Transmission system topology: Consider a connected undirected graph $\mathcal{G}=(\mathcal{V}, \mathcal{E})$ as an abstraction of transmission network of a power system. Here, $\mathcal{V}=\{1, \ldots, N\}$ is the set of nodes and $\mathcal{E} \subseteq \mathcal{V} \times \mathcal{V}$ is the set of undirected edges. We associate the nodes with the buses in the transmission network and the edges with the power lines of the network. We consider the network to consist of a set of control areas $\mathcal{A}=\left\{\mathcal{A}_{1}, \ldots \mathcal{A}_{M}\right\}$. Each control area $\mathcal{A}_{i} \in \mathcal{A}$ is defined through a set of nodes and edges, i.e. we have $\mathcal{A}_{i}=\left\{\mathcal{V}_{i}, \mathcal{E}_{i}\right\}$, where $\mathcal{V}_{i} \subseteq \mathcal{V}$ and $\mathcal{E}_{i} \subseteq \mathcal{E}$. Each node belongs to one and only one control area.

Assumption II.1 The numbering of nodes in the network is such that the first $N_{1}$ nodes belong to the control area 1, the next $N_{2}$ nodes belong to the control area 2, etc.

Remark II.2 Provision of balancing control ancillary services is performed independently within each control area in such a way that all the power imbalances which originate in one control area will eventually be compensated for by activation of ancillary services in that same control area. 
3) Nodal power injections: At a discrete time instant $k \in \mathbb{Z}$, with each node $i \in \mathcal{V}$ we associate the nodal power injection $\tilde{p}_{i}(k) \in \mathbb{R}$. Positive value of $\tilde{p}_{i}(k)$ corresponds to a flow of power into the network (production), while negative value implies that the power is extracted from the network (consumption).

4) Power flow equations: With $\tilde{\delta}_{i}(k)$ denoting the voltage phase angle at node $i \in \mathcal{V}$ and with the abbreviations $\tilde{p}(k)=$ $\operatorname{col}\left(\tilde{p}_{1}(k), \ldots, \tilde{p}_{N}(k)\right), \tilde{\delta}(k)=\operatorname{col}\left(\tilde{\delta}_{1}(k), \ldots, \tilde{\delta}_{N}(k)\right)$, the DC power flow model is given by

$$
\tilde{p}(k)=B \tilde{\delta}(k),
$$

where $B$ is the susceptance matrix. Vector of line power flows $\tilde{p}_{\mathcal{E}}(k)$ for all lines in the network can be written in the following compact form

$$
\tilde{p}_{\mathcal{E}}(k)=L \tilde{\delta}(k)
$$

where $L$ is a fixed matrix which depends on susceptances of lines and network topology.

5) Time scale separation: We distinguish two time scales, which we will conveniently call the energy time scale and the power time scale. Energy time scale is a discrete time scale with the sampling period of for example 15,30 or 60 minutes (different values can be found in different countries), which is the market trading interval for forward time markets. For convenience, we will always use the symbol $k$ to denote a time instant on the energy time scale $\left(k \in \mathbb{Z}_{+}\right)$. With the term power injection $\tilde{p}(k)$ on the energy time scale, we mean the predicted and market contracted average power injection (market contracted energy for the time interval $k$ divided by the length of the interval). Power time scale is the time scale on which real-time power balancing control loops are active and, in this paper, power time scale will be considered as continuous ${ }^{2}$ time $t \in \mathbb{R}$. Let $t=0$ be initiated at the beginning of the discrete time interval $k=0$, and define the function $\kappa: \mathbb{R}_{+} \rightarrow \mathbb{Z}_{+}$as $\kappa(t)=\left\lfloor\frac{t}{T_{s}}\right\rfloor$, where $\lfloor\cdot\rfloor$ is the floor function and $T_{s}$ is the length of the time interval on the energy time scale. If $\kappa(t)=k$, this means that the time instant $t$ on the power time scale is within the $k$-th interval on the energy time scale.

\section{PROBLEM FORMULATION}

Suppose that at time $t$ on the power time scale the system is in a steady state. Then the vector of nodal power injections $p(t)$ can be represented as $p(t)=\tilde{p}(k)+d(t)$ where $\kappa(t)=k$ and $d(t):=\operatorname{col}\left(d_{1}(t), \ldots, d_{N}(t)\right)$ is the vector of nodal power injections unpredicted in forward time (e.g., during future production scheduling on the energy time scale). The steady state power balance condition states that $\mathbf{1}_{N}^{\top} p(t)=\mathbf{1}_{N}^{\top}(\tilde{p}(k)+d(t))=0$, and since $\mathbf{1}_{N}^{\top} \tilde{p}(k)=0$ (energy markets are balanced), we have $\mathbf{1}_{N}^{\top} d(t)=0$. The vector $d(t)$ can further be decomposed into uncontrollable and controllable part, i.e. $d(t)=q(t)+f(t)$, where $q(t)$ is

\footnotetext{
${ }^{2}$ In practice, real-time power balancing controllers are in fact implemented in discrete time with the sampling time of 1 or 4 seconds. For the results of this paper, a step from the continuous time to such discrete time is straightforward.
}

the vector of uncontrollable disturbances (e.g., wind turbine productions, households loads) while the vector $f(t)$ is a vector of controllable power injections which have been brought on-line via real-time feedback loops through activation of AS control options with the goal to counteract $q(t)$. Note that in general $q(t)+f(t) \neq 0$, but $\mathbf{1}_{N}^{\top}(q(t)+f(t))=0$. Both $q(t)$ and $f(t)$ cause deviations in lines power flows from the power flows predicted during the energy market clearing ((1) and (2)). In general, if the entries in the vector $q(t)$ are sufficiency large, the new equilibrium point might be such that some lines in the transmission network are overloaded, what jeopardizes the system's integrity. Note that such situation is more likely to happen if in some location $A$ in the network there are relatively cheap AS options available in large amount, while large uncertainties and power fluctuations are characterizing some other location $B$. The AS market outcome will tend to allocate large amount of AS from location $A$. However, in some cases it might be impossible to transfer this power to the location $B$ when needed, if the transmission network power flow limits are sufficiently low. To be able to account for the reliable spatial distribution of ancillary services in the system, we need to have an appropriate model which captures the following: 1) the set of possible (or likely) values of $q(t) ; 2$ ) the relation of $f(t)$ with respect to $q(t)$.

\section{A. Uncertainties in nodal injections.}

We assume that we have the following knowledge of the uncertainties in nodal power injections. With some predefined, sufficiently high probability, we have that $q(t) \in \mathcal{Q}(k)$ for $\kappa(t)=k$, where the uncertainty set $\mathcal{Q}(k)$ is parametrised as follows

$$
\mathcal{Q}(k)=\left\{q \mid q=R(k) w, w \in \mathcal{W}(k) \subset \mathbb{R}^{m}\right\}
$$

and $0 \in \mathcal{Q}(k)$. Furthermore, the set $\mathcal{W}(k)$ is assumed to be defined as a convex hull of a finite set of vertices, that is,

$$
\mathcal{W}(k)=\operatorname{conv}\left\{w_{1}(k), \ldots, w_{T}(k)\right\},
$$

where $w_{i}(k) \in \mathbb{R}^{m}, i=1, \ldots, T$ are given.

In the remainder of the paper, we use the superscripts + and - to indicate that restoring the balance of the considered node will require positive and negative power controllable power inflow, receptively. More precisely, for negative $q_{i}(t)$, that is, for $q(t) \in\left[-r_{i}^{+}, 0\right], r^{+}>0$, nodal power balance will be restored with positive power inflow into to the node, either by direct power injection in the node, or by inflow through the connecting power lines. With this interpretation $r_{i}^{+}$can be thought of as a request for up-regulating ancillary services.

\section{B. The participation function}

The AS provision takes place on the power time scale, while the AS markets clearing is done in forward time on the energy time scale. For the AS market clearing we therefore require an appropriate energy time scale model of 
the power time scale processes. Here we will use the closedloop steady-state relations between $q$ and $f$ are a suitable model for this purpose.

Let $a_{i}^{+}(k)$ and $a_{i}^{-}(k)$ for $i=1, \ldots, N$ be respectively the amount of up-regulating and down-regulating AS options scheduled and committed at node $i$. Furthermore, let $a^{+}(k):=\operatorname{col}\left(a_{1}^{+}(k), \ldots, a_{N}^{+}(k)\right)$ and $a^{-}(k):=$ $\operatorname{col}\left(a_{1}^{-}(k), \ldots, a_{N}^{-}(k)\right)$. Suppose that the power time signal $q(t)$ is constant and that the power system is stable. Than in steady state the following holds

$$
f(t)=\gamma\left(a^{+}(k), a^{-}(k), q(t)\right)
$$

for $\kappa(t)=k$ and for some function $\gamma$. Note that in (5) the augments $a^{-}(k)$ and $a^{-}(k)$ are related to the energy time scale, while the argument $q(t)$ is related to the power time scale. Due to time scale separation, $a^{-}(k)$ and $a^{-}(k)$ are treated as constant parameters in the power time scale. We will call the function $\gamma$ the participation function, as it defines how the power injection $f_{i}(t)$ from controllable power prosumption units located at node $i$ participates in covering for the possible imbalances caused by $q(t)$. The participation function depends on the spatial distribution of scheduled AS services in the system, that is, it depends on $a^{-}(k)$ and $a^{-}(k)$. By designing the real-time balancing control loops, we have the freedom to synthesize the structure of the participation function $\gamma$ (i.e, the type/class of the function and the manner in which entries of $a^{+}(k)$ and $a^{-}(k)$ enter the function $\gamma$ ), while in the forward time AS markets we set the numerical values for $a^{+}(k)$ and $a^{-}(k)$, fully defining $\gamma$ as a function on power time scale.

\section{Robust congestion constraints}

Let $\tilde{l}(k)$ and $l(k)$ be elementwise positive vectors denoting respectively the security line flow limits for $i$ ) energy market clearing and $\mathrm{ii}$ ) real-time control for all $t$ where $\kappa(t)=k$. During the market clearing for the time interval $k$, the predicted line power flows have to be lower than $\tilde{l}(k)$, i.e, with reference to (1) and (2), the market cleared power prosumptions $\tilde{p}(k)$ have to satisfy $\tilde{p}_{\mathcal{E}}(k)=L \tilde{\delta}(k) \leq \tilde{l}(k)$. In real-time however, the line flow limits $l(k)$ apply, where $\tilde{l}(k)<l(k)$ (elementwise inequality), i.e., $L \delta(t) \leq l(k)$ for $\kappa(t)=k$ and $p(t)=B \delta(t)$. In this paper we require the real-time congestion constraint $L \delta(t) \leq l(k)$ to be satisfied for $t, \kappa(t)=k$, for which the system is in steadystate. Stated differently, for any admissible constant valued disturbance with $q(t) \in \mathcal{Q}(k)$, the power system has to have an asymptotically stable equilibrium point for which the line power flow limits $l(k)$ are not violated. Suppose that the energy market is cleared with $\tilde{p}(k)$ and corresponding $\tilde{\delta}(k)$. Let $\Delta l(k):=l(k)-L \tilde{\delta}(k)$, i.e., $\Delta l(k)$ is the additional line power flow capacity remaining after the energy market clearing.

We define the robust congestion constraints as follows. For all constant valued $q(t) \in \mathcal{Q}(k)$, the total nodal power injections $p(t)=\tilde{p}(k)+q(t)+f(t)$ on the power time scale, in steady-state, must not induce power flows which exceed the line flow limits $l(k)$. To further formalize this notion, consider first the power balance condition which states $\tilde{p}(k)+q(t)+f(t)=B(\tilde{\delta}(k)+\delta(t))$ what with (1) further implies $q(t)+f(t)=B \delta(t)$ for some $\delta(t)$. With (3) and (5), the robust congestion constraints are formalized as follows

$$
L \delta \leq \Delta l(k) \text { for all } \delta \in \mathcal{D}(k)
$$

where

$$
\mathcal{D}(k):=\left\{\begin{array}{ll}
R \mid & R(k) w+\gamma\left(a^{+}(k), a^{-}(k), R(k) w\right)=B \delta, \\
w \in \mathcal{W}(k)
\end{array}\right\},
$$

that is, $\mathcal{D}(k)$ is the set of all possible steady-state voltage phase angles when uncontrollable disturbances $q$ vary in $\mathcal{Q}(k)$ and the feedback control law implies (5) in steadystate. Note that other defining elements in (6), (7) are fixed $(l(k)$ is fixed the moment the energy market is cleared) before the AS market clearing takes place.

\section{The AS nodal pricing problem}

1) Ancillary services bids: Market agents submit the quantity-to-price bids for AS options for some future time interval $k$ on the energy time scale. We assume that each market agent specifies the location (node) of the offered AS in the bid. By aggregating all the bids related to some node $i$, the aggregated quantity-to-price up-regulating and downregulating AS bids are respectively denoted by $\beta_{i}^{+}\left(a_{i}^{+}(k)\right)$ and $\beta_{i}^{-}\left(a_{i}^{-}(k)\right)$. For $s \in\{+,-\}$, the interpretation of $\beta_{i}^{s}\left(a_{i}^{s}(k)\right)$ is as follows: if the AS price at the node $i$ would be $\lambda_{i}^{s}$, then the market agents are willing to reserve the total amounts of $\tilde{a}_{i}^{+}, \tilde{a}_{i}^{-}$of ancillary services, where $\beta_{i}^{s}\left(\tilde{a}_{i}^{s}\right)=\lambda_{i}^{s}$, $s \in\{+,-\}$.

2) Perfect competition and the AS costs: We assume conditions of perfect competition in the AS markets, see e.g. [6]. This implies that the market agents are price takers and their optimal bidding strategy is to bid the incremental AS costs, i.e., we have $\beta_{i}^{s}\left(a_{i}^{s}\right)=\frac{\partial J_{i}^{s}\left(a_{i}^{s}\right)}{\partial a_{i}^{s}}$, where $J_{i}\left(a_{i}^{s}\right)$ is the cost for allocating and providing the amount $a_{i}^{s}$ of the corresponding AS options. Therefore, from the submitted bids, we can devise the AS costs as $J_{i}^{s}\left(a_{i}^{s}\right)=\int_{\underline{a}_{i}^{s}}^{a_{i}^{s}} \beta_{i}^{s}(x) d x$, where $\underline{a}_{i}^{s}$ is the lower bound on quantity $a_{i}^{s}$ in the offered bid.

Remark III.1 Assumption of perfect competition implies that the functions $J_{i}^{s}(\cdot)$ are convex functions for all $i \in$ $\{1, \ldots, N\},[6]$.

3) Accumulation of AS options: Remark II.2 implies that to compensate for uncertainties in some control area $\mathcal{A}_{i}$, we have to accumulate amount of AS options sufficient to cover for the worst case imbalance scenario in that control area. With Assumption II.1 this requirement is formalized as follows. Let $\mathbf{e}_{\mathcal{A}_{i}}:=\operatorname{col}\left(\mathbf{0}_{\left(N_{1}+\ldots+N_{i-1}\right)}, \mathbf{1}_{N_{i}}, \mathbf{0}_{\left(N_{i+1}+\ldots+N_{M}\right)}\right)$, and let

$$
\begin{aligned}
r_{\mathcal{A}_{i}}^{+} & :=\max \left(\mathbf{e}_{\mathcal{A}_{i}}^{\top} R w_{1}, \ldots, \mathbf{e}_{\mathcal{A}_{i}}^{\top} R w_{T}\right), \quad i=1, \ldots, M \quad \text { (8a) } \\
r_{\mathcal{A}_{i}}^{-} & :=\left|\min \left(\mathbf{e}_{\mathcal{A}_{i}}^{\top} R w_{1}, \ldots, \mathbf{e}_{\mathcal{A}_{i}}^{\top} R w_{T}\right)\right|, \quad i=1, \ldots, M,(8 \mathrm{~b})
\end{aligned}
$$

where $w_{i}$ are the imbalance vertices from (3), (4). Then $r_{\mathcal{A}_{i}}^{+}$and $r_{\mathcal{A}_{i}}^{-}$denote respectively the total required amount of up-regulating and down-regulating AS services in the 
control area $\mathcal{A}_{i}$. Note that for each $i$, both $r_{\mathcal{A}_{i}}^{+}$and $r_{\mathcal{A}_{i}}^{-}$are nonnegative $\left(r_{\mathcal{A}_{i}}^{+}\right.$is nonegative since $\left.0 \in \mathcal{Q}(k)\right)$. Finally, with the abbreviations $E:=\operatorname{col}\left(\mathbf{e}_{\mathcal{A}_{1}}^{\top}, \ldots, \mathbf{e}_{\mathcal{A}_{M}}^{\top}\right)$ and $r^{s}:=$ $\operatorname{col}\left(r_{\mathcal{A}_{1}}^{s}, \ldots, r_{\mathcal{A}_{M}}^{s}\right), s \in\{+,-\}$, the requirements for $\mathrm{AS}$ accumulation are written in the following compact form

$$
E a^{+}=r^{+}, \quad E a^{-}=r^{-} .
$$

4) The pricing problem: We are now finally ready to formalize the pricing problem as follows.

Problem III.2 Let $\tilde{p}(k)$ and $\Delta l(k)$ be given after the energy market closure and let $\mathcal{Q}(k)$ be given in the form of (3), (4). Given the set of AS bids $\left\{\beta_{i}^{+}, \beta_{i}^{-}\right\}_{i \in\{1, \ldots, N\}}$ and the structure of the participation function $\gamma(\cdot, \cdot, \cdot)$, determine a vector of up-regulating AS nodal prices $\lambda^{+}(k):=$ $\operatorname{col}\left(\lambda_{1}^{+}(k), \ldots, \lambda_{N}^{+}(k)\right)$ and a vector of down-regulating AS prices $\lambda^{-}(k):=\operatorname{col}\left(\lambda_{1}^{-}(k), \ldots, \lambda_{N}^{-}(k)\right)$, so that when each market agent is committed for provision of the AS options $a_{i}^{+}=\tilde{a}_{i}^{+}, a_{i}^{-}=\tilde{a}_{i}^{-}$according to its bids, i.e., when $\beta_{i}^{+}\left(\tilde{a}_{i}^{+}\right)=\lambda_{i}^{+}, \beta_{i}^{-}\left(\tilde{a}_{i}^{-}\right)=\lambda_{i}^{-}, i=1, \ldots, N$, the following is achieved: the total AS costs $\sum_{i=1}^{N}\left(J_{i}\left(\tilde{a}_{i}^{+}(k)\right)+J_{i}\left(\tilde{a}_{i}^{-}(k)\right)\right)$ are minimizied subject to the robust congestion constraints (6), (7).

\section{THE PARTICIPATION FUNCTION}

In the remainder, we will drop the reference to the time instant $k$ on the energy time scale, as all energy time scale quantities are related to the same $k$. Having in mind Assumption II.1, let $q_{\mathcal{A}_{1}}(t):=\operatorname{col}\left(q_{1}(t), \ldots, q_{N_{1}}(t)\right)$, $q_{\mathcal{A}_{2}}(t):=\operatorname{col}\left(q_{N_{1}+1}(t), \ldots, q_{N_{1}+N_{2}}(t)\right)$, etc. Note that $q(t)=\operatorname{col}\left(q_{\mathcal{A}_{1}}(t), \ldots, q_{\mathcal{A}_{M}}(t)\right)$. Analogously, let us define $f_{\mathcal{A}_{1}}(t):=\operatorname{col}\left(f_{1}(t), \ldots, f_{N_{1}}(t)\right)$, $f_{\mathcal{A}_{2}}(t):=\operatorname{col}\left(f_{N_{1}+1}(t), \ldots, f_{N_{1}+N_{2}}(t)\right)$, etc. Then $f=\operatorname{col}\left(f_{\mathcal{A}_{1}}, \ldots, f_{\mathcal{A}_{M}}\right)$. Finally, in the same manner we define $a_{\mathcal{A}_{i}}^{+}$and $a_{\mathcal{A}_{i}}^{+}$as respectively vector of AS options of up-regulating services and down-regulating services, for the $i$-th control area. We have $\operatorname{col}\left(a_{\mathcal{A}_{1}}^{+}, \ldots, a_{\mathcal{A}_{M}}^{+}\right)=a^{+}$, $\operatorname{col}\left(a_{\mathcal{A}_{1}}^{-}, \ldots, a_{\mathcal{A}_{M}}\right)=a^{-}$. In the standard real-time control structure, each control area internally balances its power deviations by activating the AS options from within the area, that is,

$$
\mathbf{1}_{N_{i}}^{\top} f_{\mathcal{A}_{i}}(t)=-\mathbf{1}_{N_{i}}^{\top} q_{\mathcal{A}_{i}}(t) .
$$

More precisely, for each control area in steady-state we have the following relations

$$
f_{\mathcal{A}_{i}}(t)=\left\{\begin{array}{lll}
-\alpha_{\mathcal{A}_{i}}^{+}\left(a_{\mathcal{A}_{i}^{+}}\right) \overline{q_{\mathcal{A}_{i}}}(t) & \text { if } & \overline{q_{\mathcal{A}_{i}}}(t) \leq 0 \\
-\alpha_{\mathcal{A}_{i}}^{-}\left(a_{\mathcal{A}_{i}}^{-}\right) \overline{q_{\mathcal{A}_{i}}}(t) & \text { if } & \overline{q_{\mathcal{A}_{i}}}(t)>0
\end{array}\right.
$$

where $\overline{q_{\mathcal{A}_{i}}}(t):=\mathbf{1}_{N_{i}}^{\top} q_{\mathcal{A}_{1}}(t)$ is the total aggregated power deviation in the area, while $\alpha_{\mathcal{A}_{i}}^{+}\left(a_{\mathcal{A}_{i}^{+}}\right) \in \mathbb{R}^{N_{i}}$ and $\alpha_{\mathcal{A}_{i}}^{-}\left(a_{\mathcal{A}_{i}}\right) \in$ $\mathbb{R}^{N_{i}}$ are the up-regulating and down-regulating participation vector, respectively. Up-regulating participation vector is used to distribute the total uncontrollable deviation $\overline{q_{\mathcal{A}_{i}}}(t)$ over the set of controllable prosumptions, when $\overline{q_{\mathcal{A}_{i}}}(t)$ is nonpositive, that is, when there is a lack of power in the control area. Lack of power implies activation of up-regulating AS services, and therefore the participation vector $\alpha_{\mathcal{A}_{i}}^{+}\left(a_{\mathcal{A}_{i}^{+}}\right)$ is a function of $a_{\mathcal{A}_{i}}^{+}$only. Analogous holds for the downregulating participation vector $\alpha_{\mathcal{A}_{i}}^{-}\left(a_{\mathcal{A}_{i}^{-}}\right)$, which is used in case of power surplus. The $j$-th entry of a participation vector defines a fraction of total imbalance $\overline{q_{\mathcal{A}_{i}}}(t)$ that is covered by activating controllable prosumption at the $j$-th node of that control area. This fraction depends on relative contribution of (the scheduled AS options at) that node in the sum of all accumulated AS options in the control area. With the AS accumulation constraints (9), we have

$$
\alpha_{\mathcal{A}_{i}}^{+}\left(a_{\mathcal{A}_{i}^{+}}\right)=a_{\mathcal{A}_{i}}^{+} \cdot \frac{1}{r_{\mathcal{A}_{i}}^{+}}, \quad \alpha_{\mathcal{A}_{i}}^{-}\left(a_{\mathcal{A}_{i}^{-}}\right)=a_{\mathcal{A}_{i}}^{-} \cdot \frac{1}{r_{\mathcal{A}_{i}}^{-}} .
$$

With respect to (9) and (12) note that $i$-th entry of the vector $E a^{s}$ is precisely equal to $\mathbf{1}_{N_{i}}^{\top} a_{\mathcal{A}_{i}}^{s}$, and therefore $\mathbf{1}_{N_{i}}^{\top} a_{\mathcal{A}_{i}}^{s}=$ $r_{\mathcal{A}_{i}}^{s}$, where $s \in\{+,-\}$. Finally, note that by construction we have $\mathbf{1}_{N_{i}}^{\top} \alpha_{\mathcal{A}_{i}}^{s}\left(a_{\mathcal{A}_{i}^{s}}\right)=1$, what is in accordance with the balancing condition (10). In a more compact way, the relations (11) can be written in the following equivalent form

$$
f_{\mathcal{A}_{i}}(t)=-\alpha_{\mathcal{A}_{i}}^{+} \overline{q_{\mathcal{A}_{i}}^{+}}(t)-\alpha_{\mathcal{A}_{i}}^{-} \overline{q_{\mathcal{A}_{i}}^{-}}(t)
$$

where $\overline{q_{\mathcal{A}_{i}}^{+}}(t):=\min \left(\overline{q_{\mathcal{A}_{i}}}(t), 0\right)$ and $\overline{q_{\mathcal{A}_{i}}^{-}}:=\max \left(\overline{q_{\mathcal{A}_{i}}}(t), 0\right)$. Finally, for the overall network consisting of $M$ control areas we have

$$
f(t)=-\Lambda^{+}\left(a^{+}\right) \overline{q^{+}}(t)-\Lambda^{-}\left(a^{-}\right) \overline{q^{-}}(t)
$$

where $\Lambda^{+}\left(a^{+}\right):=\operatorname{diag}\left(\alpha_{\mathcal{A}_{1}}^{+}, \ldots, \alpha_{\mathcal{A}_{M}}^{+}\right), \quad \Lambda^{-}\left(\underline{\left.a^{-}\right)}:=\right.$ $\operatorname{diag}\left(\alpha_{\mathcal{A}_{1}}^{-}, \ldots, \alpha_{\mathcal{A}_{M}}^{-}\right), \overline{q^{+}}(t)=\operatorname{col}\left(\overline{q_{\mathcal{A}_{1}}^{+}}(t), \ldots, \overline{q_{\mathcal{A}_{M}}^{+}}(t)\right)$, $\overline{q^{-}}(t)=\operatorname{col}\left(\overline{q_{\mathcal{A}_{1}}^{-}}(t), \ldots, \overline{q_{\mathcal{A}_{M}}^{-}}(t)\right)$, that is, the participation function is given by

$$
\gamma\left(a^{+}, a^{-}, q(t)\right)=-\Lambda^{+}\left(a^{+}\right) \overline{q^{+}}(t)-\Lambda^{-}\left(a^{-}\right) \overline{q^{-}}(t) .
$$

The steady-state related participation function is ensured by using the standard real-time secondary control solution, see e.g. [1] for details. With $A C E_{i}(t)$ denoting the real-time measured "area control error" value of the $i$-th control area, the secondary controller which guarantees relation (13) in steady-state is given by

$$
f_{\mathcal{A}_{i}}(t)=\left\{\begin{array}{l}
-\alpha_{\mathcal{A}_{i}}^{+} k_{I} \int A C E_{i}(t) d t \text { for } \int A C E_{i}(t) d t \leq 0 \\
-\alpha_{\mathcal{\mathcal { A }}_{i}}^{-} k_{I} \int A C E_{i}(t) d t \text { for } \int A C E_{i}(t) d t>0
\end{array}\right.
$$

where $k_{I}$ is the controller gain. Alternatives to the standard real-time control structure can be found e.g., in [7], [8]. For all alternative real-time control schemes the participation function can be derived and the results of this paper can be modified (in a quite straightforward way) to solve optimal AS spatial distribution is such scenarios as well.

\section{THE NODAL PRICING SOLUTION}

With $w_{t}, t=1, \ldots, T$ defined as in (3) and (4), and with the abbreviations $q_{t}:=R w_{t}$, consider the following optimization problem, which is closely related to the pricing 
problem, i.e. to Problem III.2

$$
\min _{a^{+}, a^{-},\left\{\delta_{t}\right\}_{t \in\{1, \ldots, T\}}} \sum_{i=1}^{N}\left(J_{i}^{+}\left(a_{i}^{+}\right)+J_{i}^{-}\left(a_{i}^{-}\right)\right),
$$

subject to

$$
\begin{aligned}
& q_{t}-\Lambda^{+}\left(a^{+}\right) \overline{q_{t}^{+}}-\Lambda^{-}\left(a^{-}\right) \overline{q_{t}^{-}}=B \delta_{t}, t=1, \ldots, T \\
& L \delta_{t} \leq \Delta l, \quad t=1, \ldots, T \\
& E a^{+}=r^{+} \\
& E a^{-}=r^{-}
\end{aligned}
$$

where $\overline{q_{t}^{+}}$and $\overline{q_{t}^{-}}$are respectively determined from $\overline{q^{+}}(t)$ and $\overline{q^{-}}(t)$ by taking $q(t)=q_{t}$ (see Section IV).

Note that in the optimization problem (17) instead of the original robust congestion constraints (6) and (7), we have used the constraints (17b), (17c), which are derived from (7) evaluated at $T$ imbalances vertices. This is justified based on the following lemma.

Lemma V.1 Let $f(x, y)$ and $g(x, y)$ be linear functions in both $x$ and $y$. Then $f(\tilde{x}, y)=0, g(\tilde{x}, y) \leq 0$ hods for some $\tilde{x}$ and for all $y \in \operatorname{conv}\left\{y^{1}, \ldots, y^{N}\right\}$ if and only if $f\left(\tilde{x}, y^{i}\right)=0$, $g\left(\tilde{x}, y^{i}\right) \leq 0$ for all $i \in\{1, \ldots, N\}$.

With $\left\{\tau_{t}\right\}_{t=1, \ldots, T}, \tau_{t} \in \mathbb{R}^{N},\left\{\mu_{t}\right\}_{t=1, \ldots, T}, \mu_{t} \in \mathbb{R}^{N}$, $\sigma^{+} \in \mathbb{R}^{M}, \sigma^{-} \in \mathbb{R}^{M}$ denoting the Lagrange multipliers, the Lagrangian associated with problem (17) is

$$
\begin{aligned}
\mathcal{L} & =\sum_{i=1}^{N}\left(J_{i}^{+}\left(a_{i}^{+}\right)+J_{i}^{-}\left(a_{i}^{-}\right)\right)+\sum_{t=1}^{T} \mu_{t}^{\top}\left(L \delta_{t}-\Delta l\right) \\
& +\sum_{t=1}^{T} \tau_{t}^{\top}\left(q_{t}-\Lambda^{+}\left(a^{+}\right) \overline{q_{t}^{+}}-\Lambda^{-}\left(a^{-}\right) \overline{q_{t}^{-}}-B \delta_{t}\right) \\
& +\left(\sigma^{+}\right)^{\top}\left(E a^{+}-r^{+}\right)+\left(\sigma^{-}\right)^{\top}\left(E a^{-}-r^{-}\right) .
\end{aligned}
$$

Proposition V.2 For $t=1, \ldots, T$, let $z_{\mathcal{A}_{i}, t}^{+}:=\mathbf{1}_{N_{i}} \frac{\left(\overline{q_{\mathcal{A}_{i}, t}}\right)}{r_{\mathcal{A}_{i}}^{+}}$, $z_{\mathcal{A}_{i}, t}^{-}:=\mathbf{1}_{N_{i}} \frac{\left(\overline{q_{\mathcal{A}_{i}, t}}\right)}{r_{\mathcal{A}_{i}}^{-}}$, where $\overline{q_{\mathcal{A}_{i}, t}^{+}}$and $\overline{q_{\mathcal{A}_{i}, t}^{+}}$are respectively determined from $\overline{q_{\mathcal{A}_{i}}^{+}}(t)$ and $\overline{q_{\mathcal{A}_{i}}^{+}}(t)$ by taking $q(t)=q_{t}$ and let $z_{t}^{+}:=\operatorname{col}\left(z_{\mathcal{A}_{1}, t}^{+}, \ldots, z_{\mathcal{A}_{M}, t}^{+}\right), z_{t}^{-}:=\operatorname{col}\left(z_{\mathcal{A}_{1, t}}^{-}, \ldots, z_{\mathcal{A}_{M}, t}\right)$. Then the the optimal nodal prices for $A S$ are given as follows

$$
\lambda^{+}=-E^{\top} \tilde{\sigma}^{+}+\sum_{t=1}^{T} \tilde{\tau}_{t} \circ z_{t}^{+}, \lambda^{-}=-E^{\top} \tilde{\sigma}^{-}+\sum_{t=1}^{T} \tilde{\tau}_{t} \circ z_{t}^{-} .
$$

where $\tilde{\sigma}^{+}, \tilde{\sigma}^{-}+$and $\left\{\tilde{\tau}_{t}\right\}_{t=1, \ldots, T}$ are the optimal values of the corresponding dual variables in the Lagrange dual problem with Lagrangian defined by (18), while $\circ$ denotes the elementwise (Hadamard) product of vectors.

Proof: With assumption of perfect competition (see Remark III.1), the optimization problem (17) is convex with satisfied Slater's constraint qualification, what implies that strong duality holds. Let $j$ be a node which belongs to the $i$-th control area, i.e., $j \in \mathcal{A}_{i}$. Then a subset of the
Karush-Kuhn-Tucker (KKT) optimality conditions is given as follows (obtained from $\frac{\partial \mathcal{L}}{\partial a_{j}^{s}}=0$ )

$$
\frac{J_{j}^{s}\left(a_{j}^{s}\right)}{\partial a_{j}^{s}}+\tilde{\sigma}_{i}^{s}-\sum_{t=1}^{T} \tilde{\tau}_{t, j} \cdot \frac{\left(\overline{q_{\mathcal{A}_{i}, t}^{+}}\right)}{r_{\mathcal{A}_{i}}^{+}}=0
$$

where $\sigma_{i}^{s}$ is the $i$-th element of $\tilde{\sigma}^{s}$ and $\tilde{\tau}_{t, j}$ is the $j$-th element of $\tilde{\tau}_{t}$, while $s\{+,-\}$. Now, since $\frac{J_{j}^{s}\left(a_{j}^{s}\right)}{\partial a_{j}^{s}}=\beta_{j}^{s}\left(s_{j}^{s}\right)$ (see Section III-D.2) and since $\beta_{j}^{s}\left(s_{j}^{s}\right)=\lambda_{j}^{s}$ (see Problem III.2), we have that $\lambda_{j}^{s}=-\tilde{\sigma}_{i}^{s}+\sum_{t=1}^{T} \tilde{\tau}_{t, j} \cdot \frac{\left(\overline{q_{\mathcal{A}_{i}, t}^{+}}\right)}{r_{\mathcal{A}_{i}}^{+}}=0$. The equalities (19) are then compact expressions for the overall network of here derived price relations.

Proposition V.3 Optimal nodal prices have the same value for all nodes in the same control area (i.e., there is a unique price per control area) in case that, in optimum, line flow limits are inactive for all lines in the system and for all $q(t) \in \mathcal{Q}(k)$. If at least one line flow limit becomes active for some $q(t) \in \mathcal{Q}(k)$, prices across nodes in a control area have in general different values.

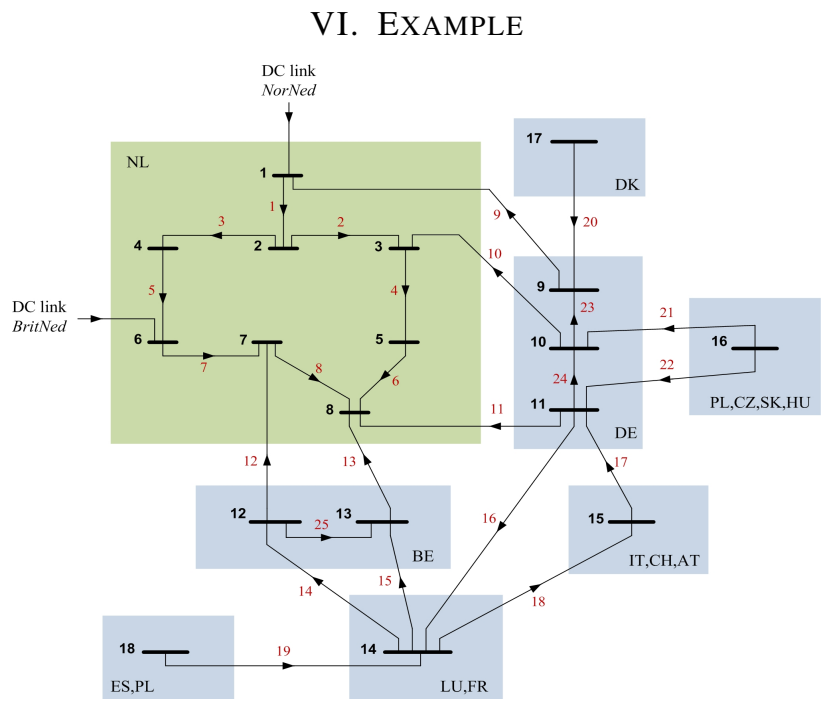

Fig. 1. Network topology.

In the presented example we have used the detailed dynamical model (with in total 64 power generating units) of the Dutch power grid, developed and verified on reallife measurements. The transmission network topology is presented in Figure 1. The Netherlands (NL) (green areain the figure) is modeled by 8 nodes and is considered as a singe control area. We present the market clearing results for both energy and AS markets, with the aim to illustrate in which way is nodal pricing in AS markets (novel notion developed in this paper) similar to the nodal pricing in energy market (well known and in practice used notion, see e.g. [9]). The results of the simulated processes on both the energy time scale and the power time scale (real-time) are presented. In real-time, the system has been constantly under influence of unpredicted changes in loads and power productions from wind turbines. The uncertainty set $\mathcal{Q}$ of 
nodal power injections has been modeled by perturbations of up to $20 \%$ of uncontrollable power prosumption at each node.

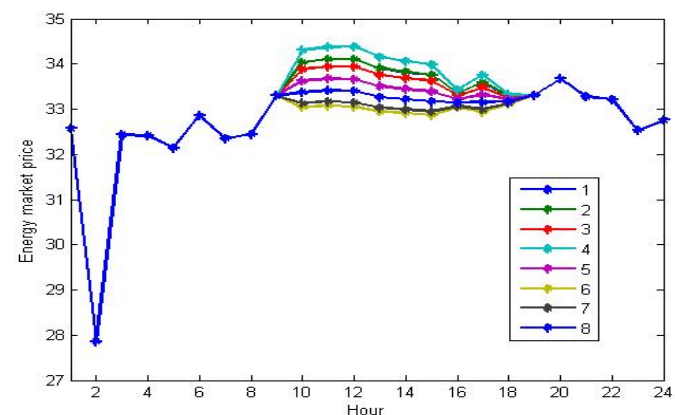

Fig. 2. Cleared energy prices (Euro/MWh) over 24 hours. Legend indicates the node numbers.

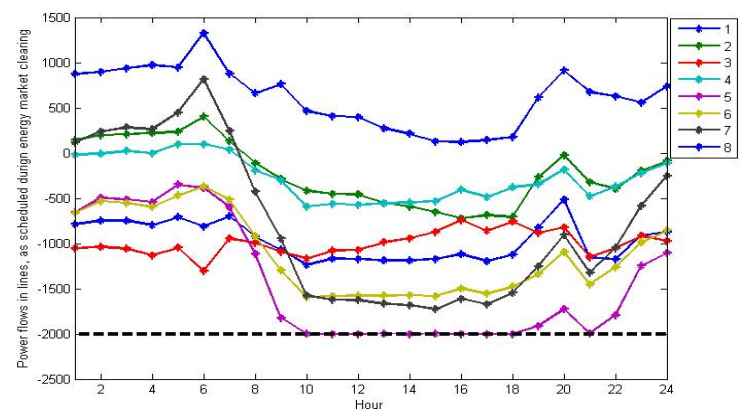

Fig. 3. Power flows (MW) in the lines ( 8 lines within NL) as scheduled during energy market clearing. Legend indicates the line numbers.

The simulated outcome of the market clearing prices for energy within the Netherlands are presented in Figure 2, while the corresponding flows in the lines are presented in Figure 3. The simulation considers period of 24 hours, with time-step on the energy time scale being one hour $(k=1, \ldots, 24)$. When there is no unique price in the system, it must be that at least one tie-line in the transmission network is at its power flow limit (nominal power flow limit $=\tilde{l}(k)$ ). In Figures 2 and 3 this can be observed for the hours 10 to 18 . In that time interval power flow in line 5 is, according to the economically optimal market clearing results, at its nominal power flow limit of $2000 \mathrm{MW}$ and there is no unique price in the system (see Figure 2). In case of AS markets, the situation is similar and described as follows. In Figure 5 we can observe power flows and the uncertainty power flow tube in the congestion critical line (line 5). Note that when, in Figure 5, the uncertainty tube touches the security line flow limit (hour 9 , i.e. around $3 \times 10^{4}$ seconds in Figure 5) we no longer have unique market clearing price for AS in the control area (see Proposition V.3), but each node in general has different AS price (Figure 4). The analogy with the energy market results (Figure 2 and 3 ) is obvious.

\section{CONCLUSIONS}

We have formulated the problem of optimal and reliable spatial distribution of power balancing ancillary services in electrical power system and have presented a solution based on a novel notion of AS nodal prices.

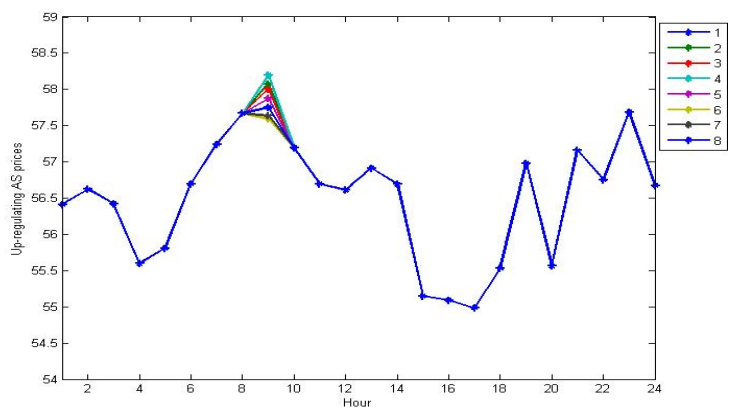

Fig. 4. Trajectory of optimal up-regulating AS prices (Euro/MWh) over 24 hours. Legend on the right indicates node numbers.

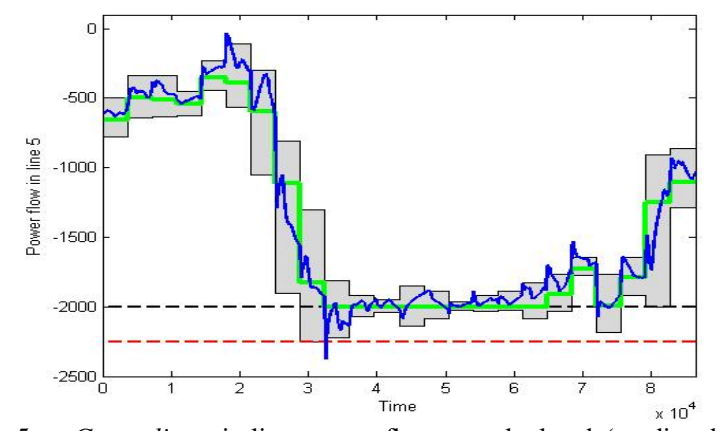

Fig. 5. Green line: tie-line power flow as calculated (predicted) during the energy market clearing. Blue line: real-time realized (simulated) power flow in the tie-line. Deviation of the blue line from the green line originates both from unpredicted changes in load/wind and from activation of AS. Gray shaded area: is the "uncertainty tube". It is the area for which we can guarantee that the corresponding tie-line power flow will remain in (possibly after a transient excursion outside that area). We guarantee that the AS control action is such that it will not allow power flows to stay outside gray area for longer time periods, or more precisely, that trajectories always converge to within the gray areas. Black dashed line: Nominal line power flow limit $=\tilde{l}(k)$ (valid and applied in the energy market clearing) of 2000 MW. Red dashed line: Security line power flow limit $=l(k)$ (valid and applied in the AS markets clearing) of $2250 \mathrm{MW}$.

\section{ACKNOWLEDGMENTS}

This work was supported by the European Commission Research Project FP7-ICT-249096 Price-based Control of Electrical Power Systems (E-Price), and by Croatian Science Foundation under the project 9354 Control of Spatially Distributed Systems. The authors would like to thank E-Price team from DNV Kema for support in the simulation case studies.

\section{REFERENCES}

[1] P. Kundur, Power System Stability and Control. McGraw-Hill, 1994.

[2] F. Alvarado, "Understanding Locational Reserves and Reliability Needs in Electricity Markets," Proc. of the HICSS'06, 2006.

[3] ENTSO-E Working Group Ancillary Services, "Position paper on crossborder balancing, ENTSO-E Tech. Rep.” 2011.

[4] ENTSO-E, "Network code on capacity allocation and congestion management, ENTSO-E Tech. Rep.” 2012.

[5] A. Jokić, "Price-based optimal control of electrical power systems," Ph.D. dissertation, TU Eindhoven, The Netherlands, 2007.

[6] S. Stoft, Power Economics. San Francisco: Ignatius Press, 2002.

[7] V. Donde, M. Pai, and I. A. Hiskens, "Simulation and optimization in an agc system after deregulation," IEEE Trans. on Power Sys., vol. 16, no. 3, pp. 481-489, 2001.

[8] Z. Zolotarev, M. Gokeler, M. Kurig, H. Neumann, and E.-M. Kurscheid, "Grid Control Cooperation: A Framework for Technical and Economical Cross-Border Optimizaztion for Load-Frequency Control," in Cigre Annual meeting 2012, 2012.

[9] F. C. Schweppe, M. C. Caramanis, and R. D. Tabors, Spot Pricing of Electricity (Power Electronics and Power Systems). Springer, 1988. 